\title{
Strategy Planning in Simultaneous Interpreting -- with Beijing Historical and Cultural Speeches as Examples
}

\author{
Hairuo Wang \\ School of Foreign Languages, North China Electric Power University, Beijing, China
}

Email address:

wanghairuo@aliyun.com

To cite this article:

Hairuo Wang. Strategy Planning in Simultaneous Interpreting -- with Beijing Historical and Cultural Speeches as Examples. International Journal of Language and Linguistics. Vol. 5, No. 5, 2017, pp. 151-160. doi: 10.11648/j.jill.20170505.15

Received: August 5, 2017; Accepted: August 30, 2017; Published: September 28, 2017

\begin{abstract}
Simultaneous interpreting contains various different layers of strategy plan at any given point of time for the practitioner. And history and culture speeches, such as the ones in China in general and in Beijing in particular, may be rather unique from the perspective of language, and may not have much "equivalence" in other cultures. It is therefore a challenge for simultaneous interpreting in message conveyance. That may mean that it is necessary to make strategy plan before the conference starts and as the conference going on in order to appropriately convey the message. This paper uses borrowed theories from psychology and management to the creation of strategy plan model of simultaneous interpreting. It attempts to find out the strategy model of simultaneous interpreting by analyzing a professional interpreter's performance working on three speeches of history and culture of Beijing and by analyzing the interview with the interpreter. Based upon the review of the literature and the analysis of the transcript of both the interpreting and the interview, a model of strategy plan of simultaneous interpreting is then proposed to facilitate the practice.
\end{abstract}

Keywords: Simultaneous Interpreting, Strategy Plan Model, Beijing Historical and Cultural Speeches

\section{Introduction}

Beijing historical and cultural speeches are considered difficult by professional interpreters, which is clearly shown in the interview with a CATTI Level $\mathrm{I}^{1}$ interpreter in this study. The interpreter, when interpreting something she was familiar with, would unconsciously make forecast on what the speaker was going to say, and thus, making a fairly adequate plan for the incoming segments of the speech, though problems do exist from time to time. However, when she was interpreting something she was less familiar with, it seems that her performance was greatly undermined by the unfamiliar concepts, which she called "words", presenting a confusion of strategies with completely different nature. Therefore, it seems that strategy plan, to a certain extent, is necessary to the interpreting of Beijing historical and cultural speeches. Therefore, this paper will focus on the discussion of strategy plan, a necessary process if the interpreting performance is to be improved.

${ }^{1}$ China Accreditation Test for Translators and Interpreters -- CATTI Level I is currently the highest level in the examination track.
Definition of several concepts should be clarified in order to have a discussion on the strategy plan in simultaneous interpreting of Beijing historical and cultural speeches. (1) Strategy plan. Strategy of interpreting is the method an interpreter chooses at the micro and macro level in order to interpret the speech. Plan is a psychological phenomenon of mental simulation of actions and inherently "adaptive activity, promoting rather than inhibiting flexible reactions to a changing environment". [5] [7: 213] (2) Simultaneous interpreting (SI). There are several definitions of simultaneous interpreting which described the practice from different perspectives. The definitions are as follows: (a) SI conveys a message into another language at virtually the same moment in time as it is expressed in the first language. [12] [23: 2] (b) SI is a unique human activity practiced under extreme cognitive processing conditions and constrained by short-term memory capacity. [1] [23: 2] (c) SI is a translation practice transferring the thoughts and ideas of one language into another and delivered almost synchronously with the speaker. [21] [23: 2] (d) SI is a type of interpreting by the interpreter which orally and precisely express the ideas and contents of one language (source language) into another (target language) 
at almost the same speed with the speaker. [22] [23: 2] All the above definitions described the features of simultaneous interpreting: (a) It is a form of interpreting. (b) The interpretation happens at virtually the same time as the original speech. (c) This type of interpretation is heavily constrained by the processing capacity of the interpreter. (3) Beijing Historical and Cultural Speeches. It is a type of speech reflecting what existed and exists in Beijing, of various aspects of history and current society of Beijing and what is rarely seen in non-Chinese cultures.

\section{Literature review}

\subsection{Strategy Planning}

Planning is conceived of as "the mental simulation of actions in a dynamic environment". [7: 214] It is psychological concept which, according to research, "can have a pervasive impact on performance, influencing not only the course of action pursued but also other, more subtle aspects of performance such as learning, motivation, and teamwork". [7] [6] [13] [18] And planning is "an inherently adaptive activity, one more likely to promote than inhibit flexible reactions to a changing environment" [7] [5], instead of an inhibiting factor to make flexible adjustment for high-level performance. [7] [14] That means that planning is aimed to better performance and it is inherently adaptive and flexible, which is in line with the nature of simultaneous interpreting where constant adjustment is needed as the interpreter deals with the unexpected for a fair amount of time in the practice. "Planning, in fact, appears to influence performance in five ways: (a) It contributes to more effective problem solving; (b) it promotes learning; (c) it enhances motivation; (d) it facilitates adaptation; and (e) it enhances coordination." [7: 225] "Planning, whatever its contributions to individual performance, is a resource-intensive activity calling for environmental appraisal, goal appraisal, contingency projection, interactive plan development, and monitoring." [7: 227] [3].

It is believed that the factors of goal identification, environmental analysis, specification of causes and consequences, forecasting, which operate together in integrated planning, play major roles in planning. [7] “...planning begins with environmental monitoring and needs assessment, followed by generation and prioritization of applicable goals. With goal identification, it becomes possible to identify the key steps and relevant contingencies needed to formulate a prototype plan. Given this prototype, key causes and contingencies operating in the local situation can be identified and the potential consequences of plan execution can be projected, leading to development of a revised, more detailed plan." [7] It seems that this is also applicable to the interpreting setting. And the "goal", which seems to be an important link in this whole planning process, may relate to what is called "skopos" in the translation theory. Skopos, proposed by Vermeer for the first time in 1970s, indicates the purpose of a piece of translation or translational behavior. [8: 78-79] [19: 71] And and the core concept of Skopotheorie is that the purpose of translational behavior is the key determinant for the translation process. [19: 71] And goal identification, or skopos identification, may be helpful to constitute the general plan of the strategy in interpreting.

Upon having a general plan, some detailed situations, or contingencies, may be seen in the execution process. And "knowledge" could be the basis for their treatment. It is believed by Mumford et al. that knowledge of the possible contingencies and that of the general rules and procedures are needed for the execution of the strategies and the dealing of the contingencies. [7] [11] It seems that interpreters should be able to grasp the knowledge on interpreting procedure and potential interpreting difficulties in order to make adequate plan for the interpreting strategy. Experienced interpreters may acquire the knowledge during the process of developing their career, while novice interpreters may do so with training and theoretical learning.

With general and local strategy plans, it is necessary to coordinate the plans at the two levels in order to make it an integral whole. It is believed that planners, which should include the interpreters, should efficiently organize their activities in accordance to the the goals, while this process may be more rapid for experts than beginners. [7: 224]

\subsection{Simultaneous Interpreting Strategy}

According to Gile [2: 156-159], there is an effort model of simultaneous interpreting, as well as several operational conditions. The simultaneous interpreting(SI) consists several parts shown in the equation:

(1) $\mathrm{SI}=\mathrm{L}+\mathrm{P}+\mathrm{M}+\mathrm{C}$

$\mathrm{L}$ stands for listening and analysis, $\mathrm{P}$ reformulation in the target language, $\mathrm{M}$ short-term memory and $\mathrm{C}$ coordination.

To make simultaneous interpreting work without failure, several operational requirements need to be met:

(2) $\mathrm{TR}=\mathrm{LR}+\mathrm{MR}+\mathrm{PR}+\mathrm{CR}$

TR Total processing capacity requirements

LR processing capacity requirements for $\mathrm{L}$

MR processing capacity requirements for $\mathrm{M}$

$\mathrm{PR}$ processing capacity requirements for $\mathrm{P}$

$\mathrm{CR}$ processing capacity requirements for $\mathrm{C}$

To make simultaneous interpreting proceed uninterruptedly, five conditions must be met at any point in the simultaneous interpreting process.

(3) $\mathrm{TR} \leq \mathrm{TA}$

TA total available processing capacity

(4) $\mathrm{LR} \leq \mathrm{LA}$

LA being the processing capacity available for $\mathrm{L}$

(5) $\mathrm{MR} \leq \mathrm{MA}$

MA being the processing capacity available for $\mathrm{M}$

(6) $\mathrm{PR} \leq \mathrm{PA}$

PA being the processing capacity available for $\mathrm{P}$

(7) $\mathrm{CR} \leq \mathrm{CA}$

$\mathrm{CA}$ being the processing capacity available for $\mathrm{C}$

The core of these inequations is that the capacity requirement for each effort should be smaller than the available. Therefore, it can be deduced that reducing the use of 
the processing capacity at any given point of time is a necessary principle for the interpreting if simultaneous interpreting is to proceed smoothly.

Many scholars believed that anticipation is an important skill in simultaneous interpreting. Jones [4: 105-107] believed that interpreters should be able to anticipate the speech in order to avoid the awkward situation of not being able to finish the sentence started which is resulted from passive following of the speech. And Nolan [9: 18-19] believed that preparation and anticipating what the speaker is going to say is very important for the interpreter. The preparation and anticipating may include getting the speech material before the conference starts, gaining familiarity with the subject matter to be discussed, and attending a meeting in advance to understand procedural rules and terms. At the same time, Nolan was aware of the potential changes in real conference setting compared with what is prepared and anticipated. Therefore, it is in line with the flexibility requirement in the strategy plan discussed in the previous part of the paper.

Nolan discussed anticipating from a general perspective, while Jones made it more concrete from several levels. Firstly, anticipating the structure and the general thrust of the speech from the context of a meeting. This is based upon the knowledge of conference and procedures, same as Nolan's proposition. Secondly, recognizing speech patterns and rhetorical structures. This is the "middle" layer of anticipation, which requires the interpreter to understand the speech type and its possible direction of development. Jones [4: 14-21] categorized four types of speeches commonly seen in international conferences, namely, reasoned logical argument, narrative speech, persuasive speech, and rhetorical speech. Wang [16] made this division of speech type a very important component of strategy for consecutive interpreting of Beijing historical and cultural speeches. Thirdly, anticipating specific words or phrases in individual sentences to understand the direction of the sentence. At the same time, Zhang believed that the communicative environment of interpretation made the operation of interpreting special, and the flexibility of the strategy of interpreting made the information between the source and the target texts asymmetrical, which is different from the traditional standard of faithfulness in translation. [10: 132] [20: 71]

\section{Research Questions and Methods}

Research questions of this study are: (1) What are challenges in interpreting of Beijing historical and cultural speeches from the interpreter's perspective? (2) What are the possible strategies adopted by the interpreter in interpreting Beijing historical and cultural speeches? (3) What is the possible model of strategy plan in simultaneous interpreting?

In order to explore the possible model of strategy plan, a piece of research is conducted to understand the possible strategy planning process of conference interpreter. And the method used in this study is qualitative research method, including observation, interview and case study and content analysis.

\subsection{Inviting an Interpreter}

As content being analyzed is based upon cases, one interpreter, who gave her full consent to the study, was invited for this study. This interpreter was female, 31 years old at the time when the research was conducted. With a master's degree in translation and interpreting and having passed CATTI level I Interpreters' Test, she got 6 years of practicing experience on the freelance conference interpreting market, having worked with hundreds of sessions of international conferences. She also worked with social institutions on the training of interpreters.

Seen from the actual performance and the interview of the interpreter in this study, it is believed that the interpreter is able to convey the message of the speaker in a general sense and made many choices based upon the consideration for the audience. Therefore, the interpreter can be confirmed as a professional one and the results in the research, to a certain extent, are able to reflect the condition of strategy plan in conference interpreting.

And because the performance and the interview with this particular interpreter seems to be representative to a certain extent, and because of the limitation of various research resources, it is decided that this study will limit the number of professional conference interpreter to only one.

\subsection{Choosing the Content of the Research}

Because of the diversity and multitude of possible speeches relevant to Beijing historical and cultural speeches, it is decided that this study will choose some representatives with the following features: (1) the content of the speech should be about Beijing history and culture; (2) the style of the speaker should be typical Chinese, which is clearly different from the speech structure and common ways of expression of normal international conferences.

It was decided to choose three speeches on TV shows, all of which being introduction of Beijing history and culture. (1) Consort kin of the imperial families, delivered by Yan Chongnian, researcher of Beijing Academy of Social Sciences, duration 37'43'. (2) Shan Jixiang guiding your to tour the Forbidden Palace, delivered by Shan Jixiang, president of the Palace Museum, duration 44'24". (3) Imperial workshop, delivered by Yan Chongnian, duration 37'49'.

The second speech differ from the first and the third in that it seemed more similar to speeches of international conference, which the speaker attended a lot. And the first and the second speeches were mainly meant for Chinese audience, thus, closer to traditional Chinese speech style. The second speech was chosen for the sake of comparison to see whether any major differences in strategy planning can be observed when the speech styles differ.

\subsection{Process of Data Collection}

There are two major steps in generating data for this study: 
(a) lab work; (b) transcription of audio and video.

The interpreter was invited to come to the lab of simultaneous interpreting with newclass DL760 system, which helps to create conditions just like the interpreter working with a real conference system. The videos of the three
TV programs were played with a computer screen, using QQ video to erase the subtitles at the bottom of the screen so that the interpreter would not be able to read the subtitles.

The processes of interpreting and interviewing can be seen from the following three figures.

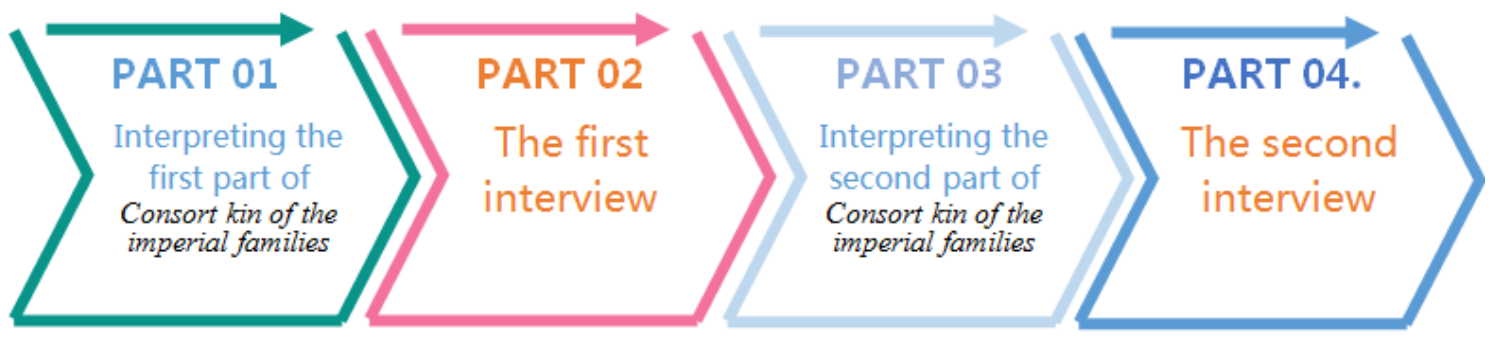

Figure 1. Data Collection of Consort kin of the imperial families.

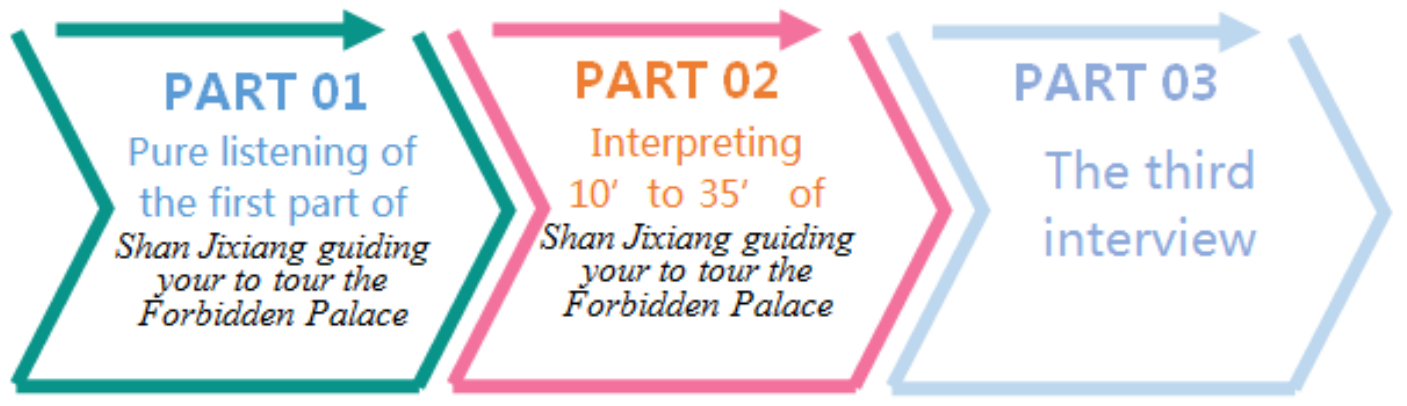

Figure 2. Data Collection of Shan Jixiang guiding your to tour the Forbidden Palace.

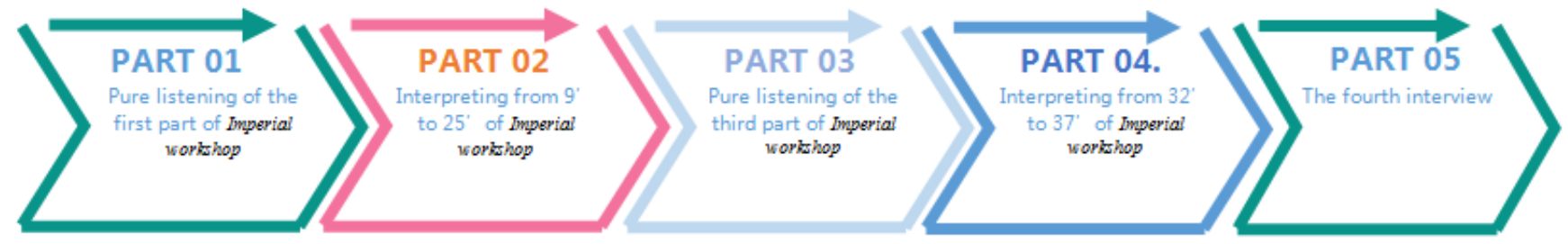

Figure 3. Data Collection of Imperial workshop.

The interpreter came with formal clothes and computer, putting herself in a state of conference. She, however, did not prepare for the topic of Consort kin of the imperial families, though the researcher primed her on the topic beforehand. This is normal circumstance during busy time of the freelance market and the researcher decided to tell her the equivalence of the key word "consort kin" in English.

After taking the data in the form of audio recording, the researcher worked with the students of the Master's program of translation and interpreting to fully transcribe the interpreting and interview.

\section{Results}

\subsection{Interview}

To understand the strategy used in the interpreter's lab performance so as to tap the potential of strategy plan in performance enhancement, interview with the interpreter is first analyzed to understand the status of the strategy used and the interpreter's perception of the strategy effect.
The framework of analysis is based upon: (1) Jones [4] proposition of the three levels of anticipation; and (2) Wang's [16] strategy model of consecutive interpreting. Therefore, three different levels of analysis on interpreting strategy are identified: (1) strategies related to the general structure and thrust of the speech which is relevant to the context. (2) strategies related to the speech type and the local sense which is beyond the words and expressions. (3) strategies related to the words and the expressions.

The four segments of the interview in this study was half-structured, with only several questions being listed as the general structure before the interview, and other questions asked during the interviewing process were spontaneous based upon points that interested the researcher. The outline of the interview consists of the following components: (1) What do you think of the difficulty level of this segment? Please make comments on the difficult points and easy points. (2) Ask questions based upon the observations of the actual performance of the interpreter, such as why certain points were dealt with in a certain way and her thought on the choice being made. (3) If it is possible, what is the interpreter's idea 
on the improvement of the strategy?

Numbers in the following three tables show the times the interpreter mentioned this point. Generally speaking, the number of times being counted is determined by the sense, for instance, the interpreter speaking a lot on one point, which will be counted as once. However, if, for instance, the interpreter mentioned two concepts or words problems consecutively, if will be counted as twice.

Table 1. Strategies of general structure and thrust of the speech.

\begin{tabular}{|c|c|c|c|c|}
\hline & $\begin{array}{l}\text { Consort kin of the imperial } \\
\text { families }\end{array}$ & $\begin{array}{l}\text { Shan Jixiang guiding your to tour } \\
\text { the Forbidden Palace }\end{array}$ & $\begin{array}{l}\text { Imperial } \\
\text { workshop }\end{array}$ & Total \\
\hline Times of mentioning in total & 7 & 8 & 2 & 17 \\
\hline Total of boxes below & 6 & 8 & 2 & 17 \\
\hline Times of passive coping or inability to deal with & 3 & 0 & 0 & 3 \\
\hline Others & 0 & 0 & 0 & 1 \\
\hline
\end{tabular}

Table 2. Strategies related to the speech type and the local sense.

\begin{tabular}{|c|c|c|c|c|}
\hline & $\begin{array}{l}\text { Consort kin of the imperial } \\
\text { families }\end{array}$ & $\begin{array}{l}\text { Shan Jixiang guiding your to tour } \\
\text { the Forbidden Palace }\end{array}$ & $\begin{array}{l}\text { Imperial } \\
\text { workshop }\end{array}$ & Total \\
\hline Times of mentioning in total & 16 & 6 & 14 & 36 \\
\hline Total of boxes below & 14 & 6 & 14 & 36 \\
\hline Times of passive coping or inability to deal with & 3 & 1 & 7 & 12 \\
\hline Others & 0 & 0 & 0 & 0 \\
\hline
\end{tabular}

Table 3. Strategies related to the words and the expressions.

\begin{tabular}{|c|c|c|c|c|c|}
\hline & $\begin{array}{l}\text { Consort } \\
\text { families }\end{array}$ & kin of the imperial & $\begin{array}{l}\text { Shan Jixiang guiding your to tour } \\
\text { the Forbidden Palace }\end{array}$ & $\begin{array}{l}\text { Imperial } \\
\text { workshop }\end{array}$ & Total \\
\hline Times of mentioning in total & 33 & & 6 & 27 & 66 \\
\hline Total of boxes below & 16 & 17 & 6 & 27 & 66 \\
\hline Times of successful strategy/actively adapting & 9 & 2 & 5 & 10 & 26 \\
\hline Times of passive coping or inability to deal with & 7 & 14 & 1 & 17 & 39 \\
\hline Others & 0 & 1 & 0 & 0 & 1 \\
\hline
\end{tabular}

Seen from the number of mentioning at the three levels of strategies, it is obvious that the total number of the strategies relevant to the general structure is smaller than those of speech type and the local sense which is smaller than those related to the words and expressions. (Hereinafter referred to as the first, second and third.) The second is $112 \%$ more than the first and the third is $83 \%$ more than the second. It may show that the interpreter paid more attention to the word and concept strategy than other two levels. Words and concepts are perhaps legitimate concerns for interpreters as these are what can be directly perceived. They are also very much important in building up the local sense. And what was mentioned quite a few times by the interpreter in her interviews of Consort kin of the imperial families and Imperial workshop was that the unfamiliar words were major obstacles for her interpreting.

Although the total number of mentioning in the second and the third is much more than that of the first, Shan Jixiang guiding your to tour the Forbidden Palace seems to be an exception. It is obvious that the interpreter talked more about the general strategy of Shan Jixiang guiding your to tour the Forbidden Palace than local sense and words and concepts. One important reason, perhaps, is because the interpreter once interpreted for the speaker on a similar speech beforehand, and was very much confident of both the general thrust and the details. Therefore, she was very happy to take the initiative to talk about the general thrust without any leading questions posed by the researcher.

On the other hand, the Consort kin and the Imperial workshop proved to be very much different. Though with questions leading to the discussion of the general thrusts from the researcher, the interpreter could not help but talked about words and concepts a lot. And she believed that not being familiar with the two topics, including the things being talked about, background knowledge, etc., was a huge constraint for her performance.

This division may mean that the interpreter is able to make the general strategy plan when she believed that the content topic being talked about was somehow "under control", while for those less familiar topics, it would be hard for her to do so. And for the unfamiliar topics, it seems that the interpreter was prone to "passive" following of the speech, instead of having an adequate strategy plan. Therefore, it is a point of discussion whether an interpreter is able to form strategy plans even on less familiar topics, maybe more on the local sense instead of the general thrust.

If we take a closer look at the two interviews of the Consort $k i n$, it is obvious that the mentioning of the first and the second level mainly appeared in the first interview, not the second one. And the second interview mainly focused on words and concepts. It may because that the interpreter believed that she was not having anything new on the strategies at the two levels, and focused only on the problems she met with in the interpreting process, which is in line with the fact that the second interview saw more times of mentioning passive coping or inability to deal with words and concepts. 


\subsection{Strategies Adopted by the Interpreter at the Three Levels}

This part is mainly based upon the analysis of the transcript of the interpreting of the three programs.

\subsubsection{Strategies of General Structure and Thrust of the Speech}

This seems to be less obvious in the actual interpreting performance, as the nature of simultaneous interpreting, unlike written translation where structure change is possible, only allows the output at the local level. Therefore, it seems difficult to observe this kind of strategy directly from the transcript, while strategies at this level may be known from the interview with the interpreter.

\subsubsection{Strategies Related to the Speech Type and the Local Sense}

(1) Consort kin of the imperial families

(a) Summary translation: Instead of following every sentence and every tiny piece of information closely, the interpreter made a summary of what the speaker has just said. This usually should be a summary of very long segment, and a summary of two or three sentences talking about one same idea is usually the longest one that may be considered acceptable.

(b) Addition: It is mainly of adding necessary explanation to the interpretation that is not in the original speech in order to locally close the gap between the two cultures.

(c) Omission: It is about leaving out the inferrable parts, summary and comments, or where the interpreter believed to be confusing to listeners if translated.

(d) Gradually adjusting interpreting sense-making: When there are some ambiguous parts, the interpreter would choose to interpret more than one possible sense at first, while gradually clarifying what was not clear at the beginning.

(e) Explanatory interpreting: It is about explaining the meaning of the speaker instead of rendering his or her words so that the listeners extract the meaning themselves.

(2) Shan Jixiang guiding your to tour the Forbidden Palace

(a) following every sentence closely: This is the most commonly used strategy of interpreters as this is the most direct way of interpreting a speech.

(b) Addition: It is mainly of adding necessary explanation to the interpretation that is not in the original speech in order to locally close the gap between the two cultures.

(c) Omission: It is about leaving out the inferrable parts, summary and comments, modifiers or where the interpreter believed to be confusing to listeners if translated.

(d) Summary translation: Instead of following every sentence and every tiny piece of information closely, the interpreter made a summary of what the speaker has just said. This usually should be a summary of very long segment, and a summary of two or three sentences talking about one same idea is usually the longest one that may be considered acceptable.

(3) Imperial workshop

(a) Combining sentences with related meanings: For example, the interpreter tended to combine the rhetorical questions and answers as they two indicate the same idea.

(b) Omission: For example, the interpreter preserved only one of the Chinese antique calendar and the Gregorian calendar, leaving out information appeared as well as summary and comment.

(c) Addition: For example, the interpreter added linkage and background information.

(d) Gradually adjusting interpreting sense-making: For example, the interpreter rendered word for word at first and then making adjustment as the sense being unfolded.

(e) Summary translation

\subsubsection{Strategies Related to the Words and the Expressions}

(1) Using explanation or superordinates to replace words not knowing equivalence

(2) Omission of unimportant words

It can be seen that there are many more types of strategies at the "mid" level than at the level of words and expressions that can be directly observed. Compared with what is mentioned in the interview, it seems that what the interpreter actually worked on is in the local levels of the "sense", which is in line with what was proposed by "theorie du sens". And although the interpreter seemed to have a lot of problems with the "words" in the interview, she actually have more tools at hand on the sense, not words, level. Or, it is possible to say that words may give interpreters problems, and she tried to solve the problem at a higher level of sense.

Whether there are strategies at the level of the general thrust and structure is not directly observable. And it may be subjected to further study to gain more understanding on it. Table 4 show the strategies at the sense level that are observed in this study.

Table 4. Strategies observed at the sense level.

\begin{tabular}{|c|c|c|}
\hline Consort kin of the imperial families & $\begin{array}{l}\text { Shan Jixiang guiding your to tour the Forbidden } \\
\text { Palace }\end{array}$ & Imperial workshop \\
\hline \multirow{6}{*}{$\begin{array}{l}\text { Summary translation } \\
\text { Addition } \\
\text { Omission } \\
\text { Gradually adjusting interpreting sense-making } \\
\text { Explanatory interpreting }\end{array}$} & Summary translation & Summary translation \\
\hline & Addition & Addition \\
\hline & Omission & Omission \\
\hline & & Gradually adjusting interpreting sense-making \\
\hline & following every sentence closely & \\
\hline & & Combining sentences with related meanings \\
\hline
\end{tabular}


As can be seen from Table 4, there are some strategies that are common to all three renditions, such as summary translation, addition, and omission, while there are some strategies more prominent in some, not others. For instance, "gradually adjusting interpreting sense-making", appeared mainly in Consort kin and Imperial workshop, which shows that probably the sense was not very clear to the interpreter at the beginning before it became clearer when sense built upon each other. It was somehow corresponds to what the interpreter said in the interview as she mentioned several times that she was not clear about certain meaning because of either lack of background knowledge or having difficulty in understanding. On the other hand, the interpreting of Shan Jixiang guiding your to tour the Forbidden Palace was much more comfortable for the interpreter as she has already interpreted for the speaker on a much similar topic before. Perhaps for the same reason, it is obvious that there is one strategy in this part of the rendition, that is, "following every sentence closely". It shows that the interpreter was at ease with the part of the rendition. And accordingly, her performance in this part is better than the other two.

\subsection{Failures in the Interpretation}

Though with certain strategies applied, there are a few failures in the interpretation of the three programs, as can be shown in the following.

\subsubsection{Consort kin of the Imperial Families}

(1) Being stuck with the dilemma of either following the sentences closely or making summary

(2) Pronoun confusions that mess the sense, for instance, the interpreter confused male and female pronouns, and sometimes used "it" without clearly referring to anything.
(3) Missing important comments

(4) Unclear rendition of human relationship, for instance, there was local confusion, and sometimes previous confusion can lead to that of later paragraphs.

(5) Unclear rendition of typical Chinese concepts, such as emotions, articles, and proper nouns.

(6) slip of tongue

(7) logical error: Sometimes there is confusion in the story.

(8) misunderstanding of source text

\subsubsection{Shan Jixiang Guiding Your to Tour the Forbidden Palace}

(1) misunderstanding of source text

(2) Unclear rendition of typical Chinese concepts such as the proper nouns.

(3) number error

(4) logical error, such as wrong addition, missing parts leading to confusion, etc.

(5) Pronoun confusions that mess the sense

\subsubsection{Imperial Workshop}

(1) Unclear rendition of human relationship

(2) Unclear rendition of typical Chinese concepts such as titles of people.

(3) number error

(4) unable to back translate foreign names

(5) logical error

(6) Grammatical errors, such as having no subject in sentences.

(7) Unclear rendition because of introduction of characters without appropriate contexts to translation listeners

(8) misunderstanding of source text

Failures in the three sections of interpreting can be summarized in Table 5.

Table 5. Failures of Interpretation.

\begin{tabular}{|c|c|c|}
\hline Consort kin of the imperial families & $\begin{array}{l}\text { Shan Jixiang guiding you to tour the } \\
\text { Forbidden Palace }\end{array}$ & Imperial workshop \\
\hline \multicolumn{3}{|l|}{$\begin{array}{l}\text { Being stuck with the dilemma of either following } \\
\text { the sentences closely or making summary }\end{array}$} \\
\hline $\begin{array}{l}\text { Pronoun confusions that mess the sense } \\
\text { Missing important comments }\end{array}$ & Pronoun confusions that mess the sense & \\
\hline Unclear rendition of human relationship & & Unclear rendition of human relationship \\
\hline $\begin{array}{l}\text { Unclear rendition of typical Chinese concepts } \\
\text { slip of tongue }\end{array}$ & Unclear rendition of typical Chinese concepts & Unclear rendition of typical Chinese concepts \\
\hline logical error & logical error & logical error \\
\hline misunderstanding of source text & $\begin{array}{l}\text { misunderstanding of source text } \\
\text { number error }\end{array}$ & $\begin{array}{l}\text { misunderstanding of source text } \\
\text { number error } \\
\text { unable to back translate foreign names } \\
\text { Grammatical errors } \\
\text { Unclear rendition because of introduction of } \\
\text { characters without appropriate contexts to } \\
\text { translation listeners }\end{array}$ \\
\hline
\end{tabular}

It is obvious from Table 5 that there are several errors all the three interpretations have in common, such as "unclear rendition of typical Chinese concepts", "logical error" and "misunderstanding of source text". It is evident that typical Chinese concepts, which describe a "world" very much different from traditional English speaking countries, could be very much different to translate unless being known previously. Therefore, it seems that much knowledge on traditional Chinese concepts seems to be a must in order to do the interpretation on related subjects. 
Logical error seems to be that at certain points, the interpreter was not able to catch up with each sentence, but was still trying to adopt the strategy of following the sentence instead of making summary, thus, causing unclear logic, which seems to be an error much more personal to the interpreter herself. Therefore, at speeches not being able to be followed, it seems the interpreter should choose either to follow closely or to make summary. To a certain extent, however, it seems that not both are usable at the same time unless the interpreter is absolutely clear of the logic she is having, which seems unlikely as the catching-up-process would consume too much energy for her already, especially when it is not possible to follow the speech closely. Therefore, choosing either of the strategy is important in this process.

At the same time, it seems that the appearance of errors is somehow related to the features of different texts. For instance, both Consort kin of the imperial families and Imperial workshop have "unclear rendition of human relationship", but not for Shan Jixiang guiding you to tour the Forbidden Palace. That shows that the first two texts are featured with complicated human relationship but not the third. It is the same with "unclear rendition because of introduction of characters without appropriate contexts to translation listeners", which only appeared in Imperial workshop. Therefore, it seems necessary that an interpreter be able to quickly identify the prominent features of the text that may affect interpreting performance and develop strategies accordingly, as flexibility and adaptability are the features of strategy plan in general and strategy plan of interpreting in particular.

And some of the errors can be more of a personal one such as "pronoun confusions that mess the sense", "slip of tongue", etc. And the strategies for these problems could be more personal as well.

It seems likely that errors could occur at the second and the third level, not the level of the general thrust, which would otherwise mean distortion of the message. Seen from the interview, it is possible that some of the errors and failures at the sense level are relevant to the fact that the interpreter was not able to adequately deal with the words and concepts. Therefore, it is deducible that in the interpretation of Beijing historical and cultural speeches, words and concepts, which carry much concrete meaning, and which are the foundations for the description of the "world", are closely linked with the "mid" structure sense-making. Therefore, it is inevitable that the interpreter needs to be able to adequately know the words and concepts, and apply strategies that could help with sense-making at the mid-level.

From the strategies and the failures, it is very clear that both are somewhat different according to the features of the texts. Therefore, it seems to be important to try to understand the features of the texts to form the appropriate strategies. At the same time, it seems that the failure and inappropriate strategies are somehow relevant to inadequate strategy plan. Therefore, it may be necessary for the interpreter to work on strategy plan if further improvement of the performance is to be made.

\section{Findings and Discussions--Strategy Plan of SI Interpreting}

With literature review and the analysis of the interview and the interpreter's performance, it is possible to know that there are several factors that compose of the strategy planning of the interpreter in the assignment.

(1) Goal identification and keeping. A Functionalist view of translation theory is that goal and function are the main factors that determine translation strategies. [15: 52] Goal identification could be of several different levels. (i) the general goal of the conference. When an interpreter serves a conference, it is important to know the theme, the background, and the purpose of the conference. (ii) the general goal of individual speakers. It is also noteworthy that every speaker may have his/her own agenda and purpose in delivering a speech, either expressing opinions, evoking emotions or communicating information, which is basically in line with the purpose of different speech types proposed by Jones [4]. This general goal obviously heavily impacts upon general strategy plan of the interpreter. For instance, if it is about evoking people's emotions, perhaps the interpreter should also try to do so as well. And if it is about communicating information, the interpreter should make sure key information is clearly conveyed by giving it high priority when the mental processing resource is not sufficient. (iii) the local purpose of the speaker. When the speakers says something, there could be a local purpose as well. For instance, in an emotional speech, there could also be important information, and the priority of then should be given to the piece of information the speaker wishes to convey, which may also be important.

The higher level goal should always be remembered when pursuing lower level goals, and when mental resource is not sufficient to fulfill all these goals, prioritization of the goals is needed for the interpreter.

Seen from the interview with the interpreter, it can be seen that the interpreter understood the goal generally to make the audience understand the sense of the speaker. However, it seems that goal identification was not particularly clear, which is understandable that the research did not take place in a real conference, and it is possible to confuse the goal. And without clearly identifying the goals, it is easy for the interpreter to fall into the "equivalence" and "loyalty", vague but popular understanding of what a piece of translation should be by many Chinese who are not translation theorists. When this happens, it is possible to see that the interpreter was trapped with not being able to follow closely while trying to, resulting in a confusion of logic.

Therefore, at the real conference setting, it seems to be a good strategy to identify goals at different levels and keep implementing the goals and prioritizing goals when they are not in line with each other.

(2) Environmental analysis and forecasting. In the strategy plan of simultaneous interpreting, environmental analysis should probably be more than the physical surrounding of the interpreter at work. In this study, it is considered to be composed of two main parts. (i) the analysis of the speaker, 
including the educational background, the profession, the delivery speed, accents, speech style, temperament, and virtually anything about the speaker that could be identified on the spot and that may affect the interpreting strategies. (ii) the analysis of the audience, including the educational background, the profession, the information reception habits, and anything that may affect the interpreting strategies.

And here, similar to goal identification and keeping, where interpreters have to prioritize goals when they may lead to competing strategies, interpreter may do the same to the strategies relevant to environmental analysis.

Forecasting is closely linked to environmental analysis in that interpreter may make forecast on what is going to be said by the speaker based upon the judgment of the speaker and the three levels of goals identified.

(3) Contingencies. It is inevitable that something unexpected may happen when interpreting. Therefore, an interpreter should always watch out for difficulties. It is, however, able to make plans on contingencies to a certain extent. (i) Being familiar with the interpreting procedure. This may relieve the interpreter of the efforts of minding the procedure, thus more processing capacity for the unexpected. (ii) Being aware of the possible difficulties. For instance, the interpreter may be aware of possible challenges in their interpreting, such as names, numbers, enumerations, a word in a foreign language, poor pronunciation, etc. [2: 160-161] Wang [17] believed that nouns (or names), numbers and logical relationship are three main difficulties for Chinese students on consecutive interpreting.

Figure 4 below is a model of Strategy Plan of Simultaneous Interpreting based upon the literature review and the case study in this research.

Model of Strategy Plan of Simultaneous Interpreting

(1) At undertaking the assignment

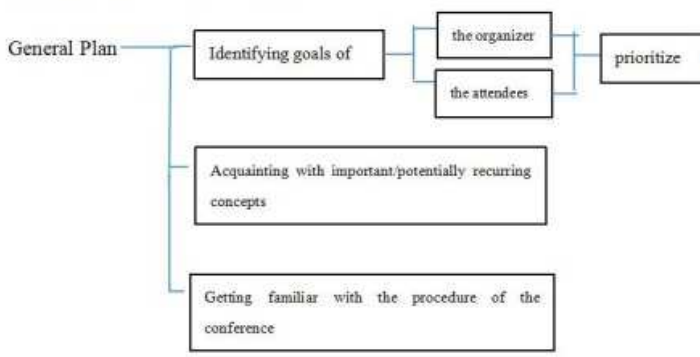

(2) At the conference venue

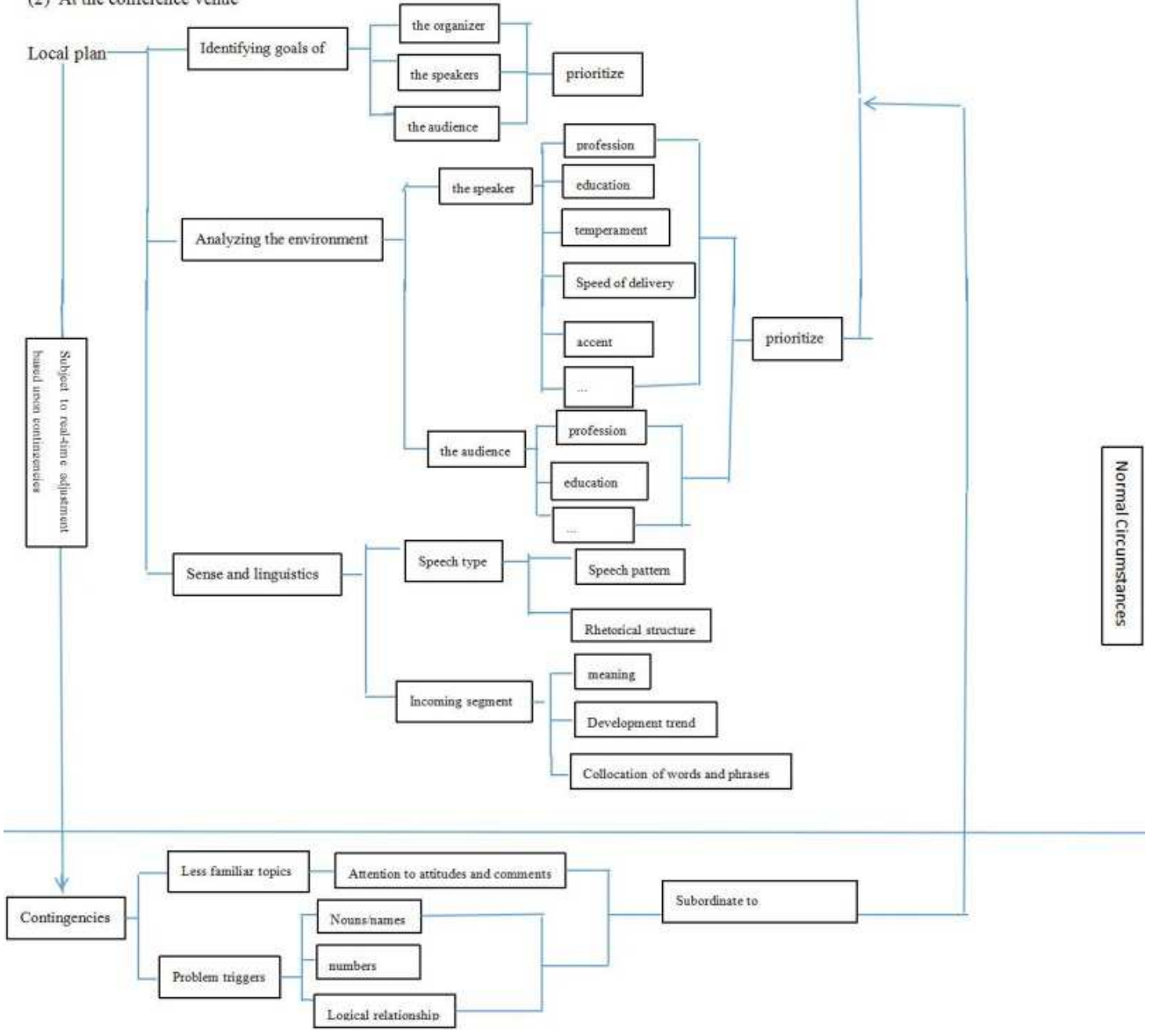

Figure 4. Model of Strategy Plan of Simultaneous Interpreting. 


\section{Conclusions}

As can be seen from the literature review and the analysis, strategy plan is important for simultaneous interpreting, as in other activities. The activity of simultaneous interpreting is one the interpreter needs to consider all relevant possible factors, which takes the utterance of the speaker as the foundation but goes far beyond that.

Strategy plan actually started from the time before the conference starts, possibly since the time of acceptance of the task, though then the plan could only be rough at best. With the preparation process going on, the plan becomes more detailed. Under "normal" circumstances, the strategy plan can be ideal, which, however, may not be "normal" circumstance in itself. The practice is full of contingencies, which has to be dealt with from time to time, as can be seen from the model. When this happens, it is the prioritization of the factors under normal circumstances that should be traced back in order to properly apply strategies to contingencies. This, together with prioritization of various different factors, demands intensive mental resource allocation as the decision-making process only takes a very short time. Therefore, the practice of simultaneous interpreting is a highly demanding one for mental processing capacity as all of the activities in the model take place at the same time while prioritization, which takes the processing to another level, taking place in just a split of a second. The internalization of the ability perhaps requires interpreter's instinct as well as training and practicing in the long while.

\section{Acknowledgements}

This study is supported by the Youth Program of Beijing Social Science Fund "an Empirical Study of Interpreting Strategies of Beijing Cultural-History Texts" (15WYC078)

Thanks to the interpreter participating this research.

\section{References}

[1] Chernov, G. V. (2011). Inference and Anticipation in Simultaneous Interpreting. Shanghai: Shanghai Foreign Language Education Press.

[2] Gile, D. (2011). Basic Concepts and Models for Interpreter and Translator Training. Shanghai: Shanghai Foreign Language Education Press.

[3] Hewes, D. E.; Graham, M. C.; Monsour, M.; Doelger, J. A. (1989). Cognitions and social information-gathering strategies: Reinterpretation assessment in second-guessing. Human Communication Research, 16(2), 297-321.

[4] Jones, R. Conference Interpreting Explained. (2008). Shanghai: Shanghai Foreign Language Education Press.

[5] Keane, M. T. (1996). On adaptation in analogy: Tests of pragmatic importance and adaptability in analogical problem solving. Quarterly Journal of Experimental Psychology, 49(49), 1062-1085.
[6] Kops, C, \& Belmont, I. (1985). Planning and organizing skills of poor school achievers. Journal of Learning Disabilities, $18(1), 8-14$.

[7] Mumford, M. D.; Schultz, R. A.; Van Doorn, J. R. (2001). Performance in Planning: Processes, Requirements, and Errors. Review of General Psychology, 5(3), 213-240.

[8] Munday, J. (2010). Introducing Translation Studies: Theories and Applications. Shanghai: Shanghai Foreign Language Education Press.

[9] Nolan, J. (2008). Interpretation: Techniques and Exercises. Shanghai: Shanghai Foreign Language Education Press.

[10] Pöchhacker, F. (2004). Introducing Interpreting Studies. London and New York: Routledge, 2004.

[11] Read, S. J. (1987). Constructing causal scenarios: A knowledge structure approach to causal reasoning. Journal of Personality and Social Psychology, 52(2), 288-302.

[12] Seleskovitch, D.; Lederer, D. (2007). Pédagogie raisonnée de l'interprétation. Beijing: China Translation and Publishing Corporation.

[13] Smith, K. G.; Locke, E. A.; Barry, D. (1990). Goal setting, planning, and organizational performance. Organizational Behavior and Human Decision Processes, 46(1), 118-134.

[14] Sternberg, R. J.; Lubart, T. I. (1996). Investing in creativity. American Psychologist, 51(7), 677-688.

[15] Wang, B. H. (2012). From Criteria of Interpreting to Norms in Interpreting--On the Construction of Interpreting Assessment Models. Shanghai Journal of Translators, 3, 49-54.

[16] Wang, H. R. (2016). A Study on the Strategy of Chinese-English Consecutive Interpreting of Beijing Historical and Cultural Speeches. International Journal of Applied Linguistics and Translation, 2(5), 43-52.

[17] Wang, H. R. (2015). Error Analysis in Consecutive Interpreting of Students With Chinese and English Language Pairs. Canadian Social Science, 11(11), 65-79.

[18] Weldon, E.; Jehn, K. A.; Pradhan, P. (1991). Processes that mediate the relationship between group goals and improved group performance. Journal of Personality and Social Psychology, 61(4), 555-569.

[19] Zhang, M. F. (2015). Functional Approaches to English-Chinese Translation. Beijing: Foreign Languages Press.

[20] Zhang, W. (2015). Tagging of interpreting strategies in CILC: method and significance. Journal of Foreign Languages, 38(5), 63-73.

[21] Zhang, W. W. (2011). English-Chinese Simultaneous Interpretation. Shanghai: Shanghai Foreign Language Education Press.

[22] Zhong, W. H. (2008). A Coursebook for Simultaneous Interpreting Between English and Chinese. Beijing: Higher Education Press.

[23] Zhong, W. H.; Zhan, C. (2009). A Coursebook of Simultaneous Interpreting. Beijing: Foreign Language Teaching and Research Press. 